\title{
EDITORIAL
}

\section{THE TREPONEMAL IMMOBILIZATION TEST}

Few will deny that interest in the treponemal immobilization (TPI) test is increasing. This may well be due to the fact that, at long last, we have a serological test for syphilis which employs a specific antigen. Such a test naturally gives rise to the hope that the problem of identifying non-treponemal reactors is well on the way to solution. In addition to the increasing urgency of this problem (particularly when antenatal patients are involved) information has been somewhat slow to come to hand. During the last few years there has been a steady stream of papers on the TPI test, but so many of these are concerned with relatively small numbers of sera from selected patients. Critical reviews of the literature on this subject are few and Zellmann's* no doubt is the latest and best of these.

Surprising as it may seem, relatively few sera from healthy non-treponemal patients have been tested by means of this reaction. On the other hand, much attention has been devoted to the study of the results of the TPI test with sera from patients labelled as latent syphilitics. Until recently, these patients were diagnosed by means of the standard serum tests for syphilis, but with the advent of the TPI test, many such diagnoses became suspect, and it is pertinent to ask if one is to accept the TPI reaction with such sera as 100 per cent. specific. Few workers have claimed such omniscience for

* Zellmann, H. E. (1954), Amer. J. Syph. 38, 506. any pathological test and, at this stage, the cautious may be forgiven for entertaining a degree of agnosticism whilst wondering whether those who would consign all serological tests for syphilis to the dustbin are not overstating their case.

$\mathrm{Be}$ that as it may, there is no doubt that the TPI test is a most valuable addition to our methods of diagnosing syphilis, and it seems that clinicians in Great Britain are being deprived of considerable help while the present restrictions on its employment are in force.

It is suggested, however, that more information concerning the test is required. Of necessity, evidence is slow to accumulate. This is due not only to the time factor of the technique but also to the paucity of certain types of patients (at least in Great Britain) whose sera would be suitable for investigation by means of serial testing. In addition, defaulting patients and a shifting population play their part in holding up the final assessment of the test.

Readers of this Journal will, therefore, we feel sure, welcome the articles in this issue recording the experiences of the two authors with this test. The information contained therein will add several more pieces to the jigsaw of knowledge necessary before the TPI test finally takes its rightful place in the battery of diagnostic serological tests for syphilis. 